\title{
ORIGINAL ARTICLE \\ The International Spinal Cord Injury Pain Basic Data Set (version 2.0)
}

\author{
E Widerström-Noga ${ }^{1,2}$, F Biering-Sørensen ${ }^{3}$, TN Bryce ${ }^{4}$, DD Cardenas ${ }^{2}$, NB Finnerup ${ }^{5}$, MP Jensen ${ }^{6}$, \\ JS Richards ${ }^{7}$ and PJ Siddall ${ }^{8,9}$
}

Objectives: To revise the International Spinal Cord Injury Pain Basic Data Set (ISCIPBDS) based on new developments in the field and on suggestions from the spinal cord injury $(\mathrm{SCI})$ and pain clinical and research community.

Setting: International.

Methods: The ISCIPBDS working group evaluated suggestions regarding the utility of the ISCIPBDS and made modifications in response to these and to significant developments in the field. The revised ISCIPBDS (version 2.0) was reviewed by members of the Executive Committee of the International SCl Standards and Data Sets, the International Spinal Cord Society (ISCoS) Executive and Scientific Committees, the American Spinal Injury Association and American Pain Society Boards and the Neuropathic Pain Special Interest Group of the International Association for the Study of Pain, individual reviewers and societies and the ISCoS Council.

Results: The ISCIPBDS (version 2.0) is significantly shortened but still contains clinically relevant core questions concerning $\mathrm{SCl}$-related pain. The revisions include an updated $\mathrm{SCl}$ pain classification, omission of three questions regarding temporal pain pattern and three pain interference questions. The remaining three pain interference questions concern perceived interference with activities, mood and sleep for overall pain rather than for individual pain problems and are scored on a 0 to 10 scale.

Spinal Cord (2014) 52, 282-286; doi:10.1038/sc.2014.4; published online 28 January 2014

Keywords: Spinal cord injuries; pain; pain measurement; psychometrics; pain classification; pain interference

\section{INTRODUCTION}

After the International Spinal Cord Injury Pain Basic Data Set (ISCIPBDS; version 1.1) was published in $2008^{1}$ there have been several suggestions from the clinical spinal cord injury (SCI) field to shorten it in order to increase its clinical utility. In addition, there has been a significant update of the SCI pain taxonomy. ${ }^{2,3}$ For these reasons, the ISCIPBDS working group decided to review and update the current version.

Both the original and the revised ISCIPBDS (version 2.0) are intended to be collected by health-care professionals familiar with SCI, and to be used at the initial evaluation as well as at regular follow-up sessions. Data should be collected by interview and each question read to the patient or research participant as worded.

The overall purpose of the ISCIPBDS (version 2.0) is unchanged and consistent with the purpose and vision of the International Spinal Cord Injury Data Sets. ${ }^{4}$ The ISCIPBDS should be used together with the International SCI Core Data Set, ${ }^{5}$ which includes information on date of birth and injury, gender, the cause of the SCI and neurological status including positive and negative sensory signs.

\section{MATERIALS AND METHODS}

The development of the original ISCIPBDS and information regarding the members of the working group was described in detail previously. ${ }^{1}$

The data collected provide a minimal amount of standardized information necessary for determining interference of pain with physical and emotional function and sleep, probable pain diagnosis, location, intensity and duration. In order to ensure consistency in data collection and to facilitate interpretation, detailed information is provided in the updated syllabus (version 2.0) for each variable and response category.

The development of the revised version of the ISCIPBDS (version 2.0) followed the same steps as the original version:

1. The working group made the revisions as described below via e-mail discussions. A subset of the working group also had a face-to-face meeting during the American Spinal Injury Association (ASIA) conference in May 2013 in Chicago. The version resulting from this meeting was further developed via e-mail correspondence.

2. The ISCIPBDS (version 2.0) was reviewed by members of the Executive Committee of the International SCI Standards and Data Sets. The suggestions from the Committee members were discussed in the working group and appropriate changes were made.

${ }^{1}$ Miami Project to Cure Paralysis, Lois Pope Life Center, Miller School of Medicine, University of Miami, Miami, FL, USA; ${ }^{2}$ Department of Rehabilitation Medicine, Miller School of Medicine, University of Miami, Miami, FL, USA; ${ }^{3}$ Clinic for Spinal Cord Injuries, Glostrup University Hospital, and Faculty of Health and Medical Sciences, University of Copenhagen, Copenhagen, Denmark; ${ }^{4}$ Department of Rehabilitation Medicine, Icahn School of Medicine at Mount Sinai, New York, NY, USA; ${ }^{5}$ Danish Pain Research Center, Aarhus University Hospital, Aarhus, Denmark; ${ }^{6}$ Department of Rehabilitation Medicine, University of Washington, Seattle, WA, USA; ${ }^{7}$ Department of Physical Medicine and Rehabilitation, University of Alabama at Birmingham, Birmingham, AL, USA; ${ }^{8}$ Department of Pain Management, Greenwich Hospital, Hammond Care, Sydney, New South Wales, Australia and 9 Sydney Medical School-Northern, University of Sydney, Sydney, New South Wales, Australia

Correspondence: Dr E Widerström-Noga, Miami Project to Cure Paralysis, Lois Pope Life Center, Miller School of Medicine, University of Miami, PO Box 016906 (R-48), Miami, FL 33101, USA.

E-mail: ewiderstrom-noga@med.miami.edu

Received 12 November 2013; revised 11 December 2013; accepted 3 January 2014; published online 28 January 2014 
3. The ISCIPBDS (version 2.0) was reviewed by members of the International Spinal Cord Society (ISCoS) Executive and Scientific Committees and ASIA Board with no further suggestions for improvement.

4. The ISCIPBDS (version 2.0) was reviewed and endorsed by members of the American Pain Society (APS) Board and the Neuropathic Pain Special Interest Group (NeuPSIG) of the International Association for the Study of Pain (IASP).

5. Organizations, societies and individuals with an interest in SCI-related pain were invited to review and comment on the ISCIPBDS (version 2.0). The data set was also posted on the ISCoS and ASIA websites to allow additional comments and suggestions.

6. To finalize the ISCIPBDS (version 2.0), members of the ISCoS Executive and Scientific Committees and the ASIA Board reviewed the data set for final approval.

\section{RESULTS}

Several changes were made from version 1.1 to version 2.0. These include: (1) omission of some items; (2) updating the SCI pain classification; and (3) Re-structuring of the ISCIPBDS. These changes (detailed below) were made because of both recent updates to the pain classification scheme and suggestions from the field to shorten and focus the ISCIPBDS to facilitate its clinical utility.

\section{Omission of items}

The ISCIPBDS was shortened to contain only those items that we believe essential for a correct pain classification and for determining intensity and broad impact on activities, sleep and mood. Items that did not address these critical factors were removed.

The variables 'Number of days with pain in the last 7 days including today', 'How long does your pain usually last?' and 'When is the pain most intense?', were removed as these were not considered critical for determining pain type, intensity and pain impact. Moreover, in a validation study of the ISCIPBDS, we found little support for the validity of the two pain duration items (days of pain and duration of pain incidents ${ }^{6}$ ). The findings from our study influenced our decision to remove these items.

The following three interference items were removed: 'How much do you limit your activities in order to keep your pain from getting worse?', 'How much has your pain changed your ability to take part in recreational and other social activities?' and 'How much has your pain changed the amount of satisfaction or enjoyment you get from family-related activities?' In the validation study cited above, the internal consistency (Cronbach's alpha) suggested some redundancy among the pain interference items and therefore we concluded that all six items were not required to adequately assess the pain interference domain. In our study, ${ }^{6}$ we found that the three remaining interference items showed the strongest associations with the validity criteria. We concluded from these findings that only three items were needed to provide a valid measure of pain interference.

Another effort to shorten the ISCIPBDS included the instruction to apply the pain interference items to overall pain rather than to each of the different pain problems. Moreover, in order to make these items consistent with the pain intensity ratings, we changed the response levels from 0 to 6 to the more common 0 to 10 numerical rating scale (NRS) metric.

\section{Updated SCI pain classification}

Our proposed changes include updating the pain classification to be consistent with the new SCI pain taxonomy that has been published and validated. ${ }^{2,3}$
Related to the variable Type of pain, an extra option 'Other' was added. In addition, only one choice of pain type can be made on the form. The codes include:

Musculoskeletal (nociceptive)

Visceral (nociceptive)

Other (nociceptive)

At-level SCI (neuropathic)

Below-level SCI (neuropathic)

Other (neuropathic)

Other

Unknown

These types of pain should be coded using the following criteria:

Musculoskeletal (nociceptive). Musculoskeletal (nociceptive) pain refers to: (1) pain in a region with preserved sensation above, at or below the neurological level of injury (NLI) and (2) pain believed to be originating in musculoskeletal structures. This pain type is indicated by pain with a dull or aching quality, pain initiated or aggravated by movement, tenderness of musculoskeletal structures on palpation, relief in response to anti-inflammatory or opioid medications, or evidence of skeletal pathology on imaging consistent with the pain presentation. Examples include: mechanical pain, spinal fractures, muscular injury, shoulder overuse syndromes and muscle spasm. $^{7-10}$

Visceral (nociceptive). Visceral (nociceptive) pain refers to: (1) pain located in the thorax, abdomen or pelvis; and (2) pain believed to originate in visceral structures. This pain type is indicated by dull, tender or cramping pain and related to visceral pathology or dysfunction, such as infection or obstruction, for example, urinary tract infection, ureteric calculus and constipation. ${ }^{2,7-10}$

Other (nociceptive). Other (nociceptive) pain refers to nociceptive pains that do not fall into the musculoskeletal or visceral categories directly related or unrelated to SCI, for example, pain associated with ulceration of the skin and headache. ${ }^{9}$

At-level SCI (neuropathic). At-level SCI (neuropathic) pain refers to neuropathic pain distributed segmentally due to spinal cord or nerve root damage. At-level neuropathic pain is located unilaterally or bilaterally anywhere within the dermatome of the NLI and three dermatomes below this level. This pain is often perceived as hotburning, tingling, pricking, pins and needles, squeezing, cold, electric or shooting and often associated with allodynia, hypoalgesia or hyperalgesia within the painful area.

Below-level SCI (neuropathic). Below-level SCI (neuropathic) pain refers to neuropathic pain that is located more than three dermatomes below the NLI and may be perceived up to the NLI. This pain is often perceived as hot-burning, tingling, pricking, pins and needles, squeezing, cold, electric or shooting and often associated with allodynia, hypoalgesia or hyperalgesia within the painful area.

Other (neuropathic). Other (neuropathic) pain refers to neuropathic pains that are located above, at, or below the NLI but are not directly related to the SCI (for example, post-herpetic neuralgia, painful diabetic neuropathy, central post-stroke pain, and compressive mononeuropathies). ${ }^{8,9}$

Other. Other pain refers to: (1) pain that is unrelated to the SCI and (2) pain without any identifiable noxious stimulus, inflammation or damage to the nervous system, for example, Complex Regional Pain 
Syndrome type I, interstitial cystitis pain, irritable bowel syndrome pain and fibromyalgia.

Unknown. Unknown pain refers to pain of unknown etiology.

\section{Restructuring of the ISCIPBDS}

In addition to deletion of some items, we changed the order of the items (see Appendix). Because the three Pain Interference questions refer to 'overall pain', these items will be asked before any questions regarding specific pain types. Moreover, respondents are asked to rate the interference items on a 0 to 10 scale instead of 0 to 6 scale to be consistent with the pain intensity item. Please note that the psychometric properties were evaluated with these items scored between 0 and $6 .{ }^{6}$ However, we expect minimal to no effects on these properties with the revision.

The remaining variables listed below are unchanged and described as previously. ${ }^{1}$

Date of data collection

Have you had any pain during the last 7 days including today?

How many different pain problems do you have?

Description of the three worst pain problems

Location of pain

Average pain intensity in the last week

Date of onset

Are you using or receiving any treatment for your pain problem?

The ISCIPBDS (version 2.0) data sheet is included in the Appendix and the data sheet and the revised syllabus are available on the website of ISCoS (http://www.iscos.org.uk). Revised training cases are likewise available together with the syllabus on the ISCoS website.

\section{DISCUSSION}

The revised ISCIPBDS (version 2.0) presented in this paper is an effort to provide an abbreviated, updated and standardized way of evaluating and reporting the diverse pains that occur in persons with SCI. The ISCIPBDS has been significantly shortened, but assesses similar domains as the original version. ${ }^{1}$ The revised version presented in this paper was designed to contain a minimum most critical clinically relevant pain information that can be collected in the daily practice of health-care professionals with expertise in SCI. Another criterion is that the evaluation should be logistically feasible across various settings and countries including the developing countries. The ISCIPBDS (version 2.0) should, as the original version, be used in combination with the ASIA Impairment Scale ${ }^{11}$ to determine the extent of neurological injury and to determine the location of pain relative to injury (that is, above, at and below).

In summary, the ISCIPBDS (version 2.0) is intended to be used to evaluate pain in daily clinical practice at both in- and out-patient SCI clinics around the world. In a specialized pain clinic managing patients with SCI, more sophisticated methods including quantitative sensory assessments would add diagnostic precision.

Although one might consider the revised ISCIPBDS to have less content validity, because the revised version contains fewer items, our view is that it has retained the items that are most critical. Of more importance, perhaps, the Data Set is now more brief, which will likely increase its clinical utility. It is our intention to continue to update and improve the ISCIPBDS and the working group (through the corresponding author) welcomes additional suggestions and comments for further improvement. In particular, we welcome feedback from clinical practitioners regarding the utility of the ISCIPBDS in clinical settings.

\section{DATA ARCHIVING}

There were no data to deposit.

\section{CONFLICT OF INTEREST}

The authors declare no conflict of interest.

\section{ACKNOWLEDGEMENTS}

We thank the ISCoS, the ASIA and the APS Boards and the IASP NeuPSIG for endorsement. We also thank Lawrence Vogel and Marcel Post for helpful suggestions.

Pfizer Corp supported the initial work for development of the ISCIPBDS (version 1.1) with an unconditional educational grant.

1 Widerström-Noga E, Biering-Sørensen F, Bryce T, Cardenas DD, Finnerup NB Jensen MP et al. The International Spinal Cord Injury Pain Basic Data Set. Spinal Cord 2008; 46: 818-823.

2 Bryce TN, Biering-Sorensen F, Finnerup NB, Cardenas DD, Defrin R, Lundeberg T et al. International spinal cord injury pain (ISCIP) classification: part I. Background and description. Spinal Cord 2012; 50: 413-417.

3 Bryce TN, Biering-Sorensen F, Finnerup NB, Cardenas DD, Defrin R, Ivan E et al. International spinal cord injury pain (ISCIP) classification: part 2. Initial validation using vignettes. Spinal Cord 2012; 50: 404-412.

4 Biering-Sørensen F, Charlifue S, DeVivo M, Noonan V, Post M, Stripling T et al International spinal cord injury data sets. Spinal Cord 2006; 44: 530-534.

5 DeVivo M, Biering-Sørensen F, Charlifue S, Noonan V, Post M, Stripling T et al. International Spinal Cord Injury Core Data Set. Spinal Cord 2006; 44: 535-540.

6 Jensen MP, Widerström-Noga E, Richards JS, Finnerup NB, Biering-Sørensen F, Cardenas DD. Reliability and validity of the International Spinal Cord Injury Basic Pain Data Set items as self-report measures. Spinal Cord 2010; 48: 230-238.

7 Donovan WH, Dimitrijevic MR, Dahm L, Dimitrijevic M. Neurophysiological approaches to chronic pain following spinal cord injury. Paraplegia 1982; 20: $135-146$.

8 Siddall PJ, Yezierski RP, Loeser JD. Pain following spinal cord injury: Clinical features, prevalence, and taxonomy. IASP Newslett 2000; 3: 3-7.

9 Bryce TN, Ragnarsson KT. Epidemiology and classification of pain after spinal cord injury. Top Spinal Cord Inj Rehabil 2001; 7: 1-17.

10 Cardenas DD, Turner JA, Warms CA, Marshall HM. Classification of chronic pain associated with spinal cord injuries. Arch Phys Med Rehabil 2002; 83: 1708-1714.

11 Kirshblum SC, Burns SP, Biering-Sorensen F, Donovan W, Graves DE, Jha A et al. International standards for neurological classification of spinal cord injury (Revised 2011). J Spinal Cord Med 2011; 34: 535-546. 


\section{INTERNATIONAL SPINAL CORD INJURY PAIN BASIC DATA SET}

\section{DATA COLLECTION FORM - Version 2.0}

Date of data collection: YYYY/MM/DD

Have you had any pain during the last seven days including today?

$$
\text { No Yes }
$$

If yes:

Please note that the time period during the last week applies to all pain interference questions.

In general, how much has pain interfered with your day-to-day activities in the last week?

No interference $0-1-2-3-4-5-6-7-8-9-10$ Extreme interference

In general, how much has pain interfered with your overall mood in the last week?

No interference $0-1-2-3-4-5-6-7-8-9-10$ Extreme interference

In general, how much has pain interfered with your ability to get a good night's sleep?

No interference $0-1-2-3-4-5-6-7-8-9-10$ Extreme interference

How many different pain problems do you have?

$$
1 ; \quad 2 ; 3 ; \quad 4 ; \geqslant 5
$$

Please describe your three worst pain problems:

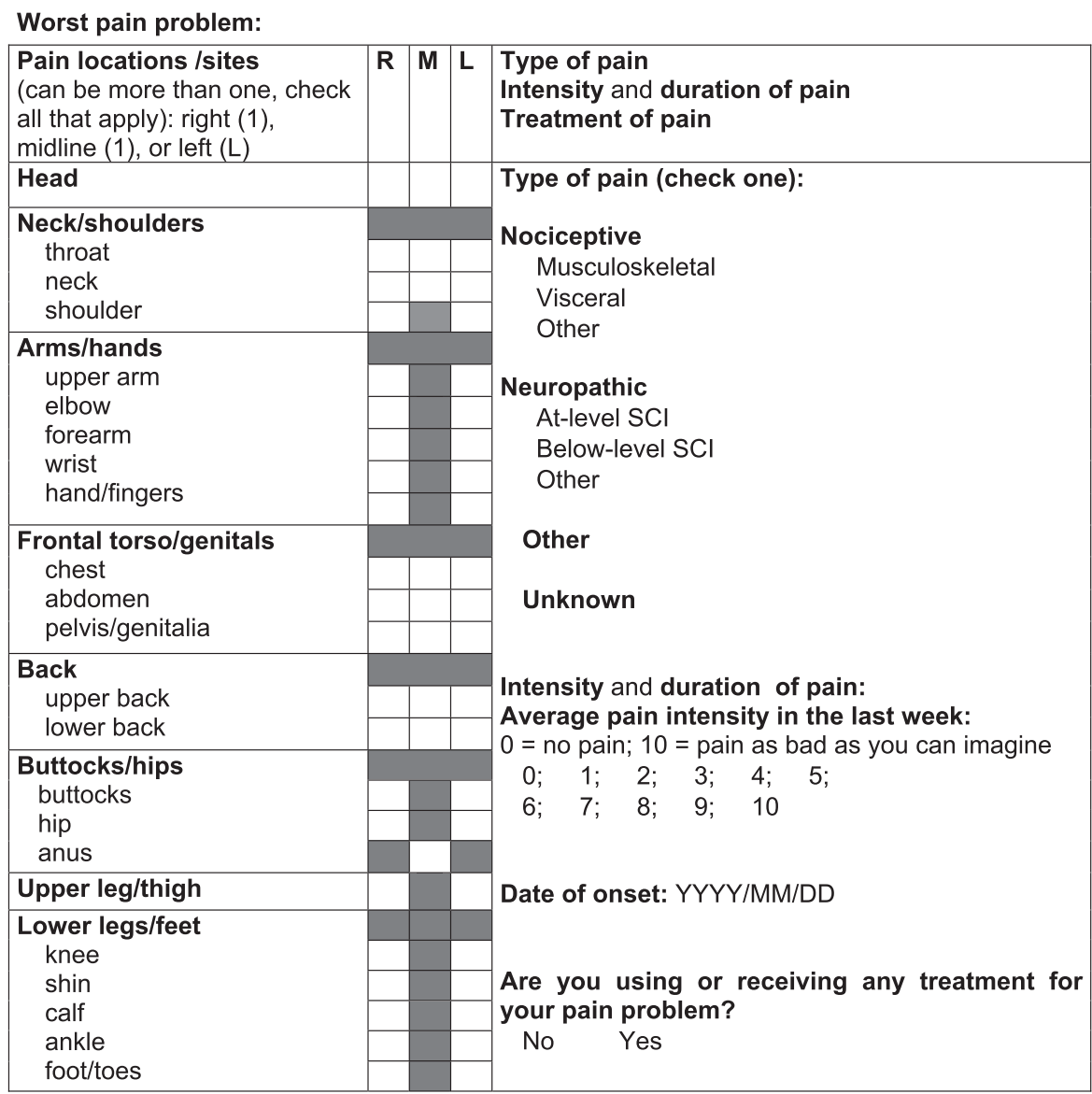


Second worst pain problem:

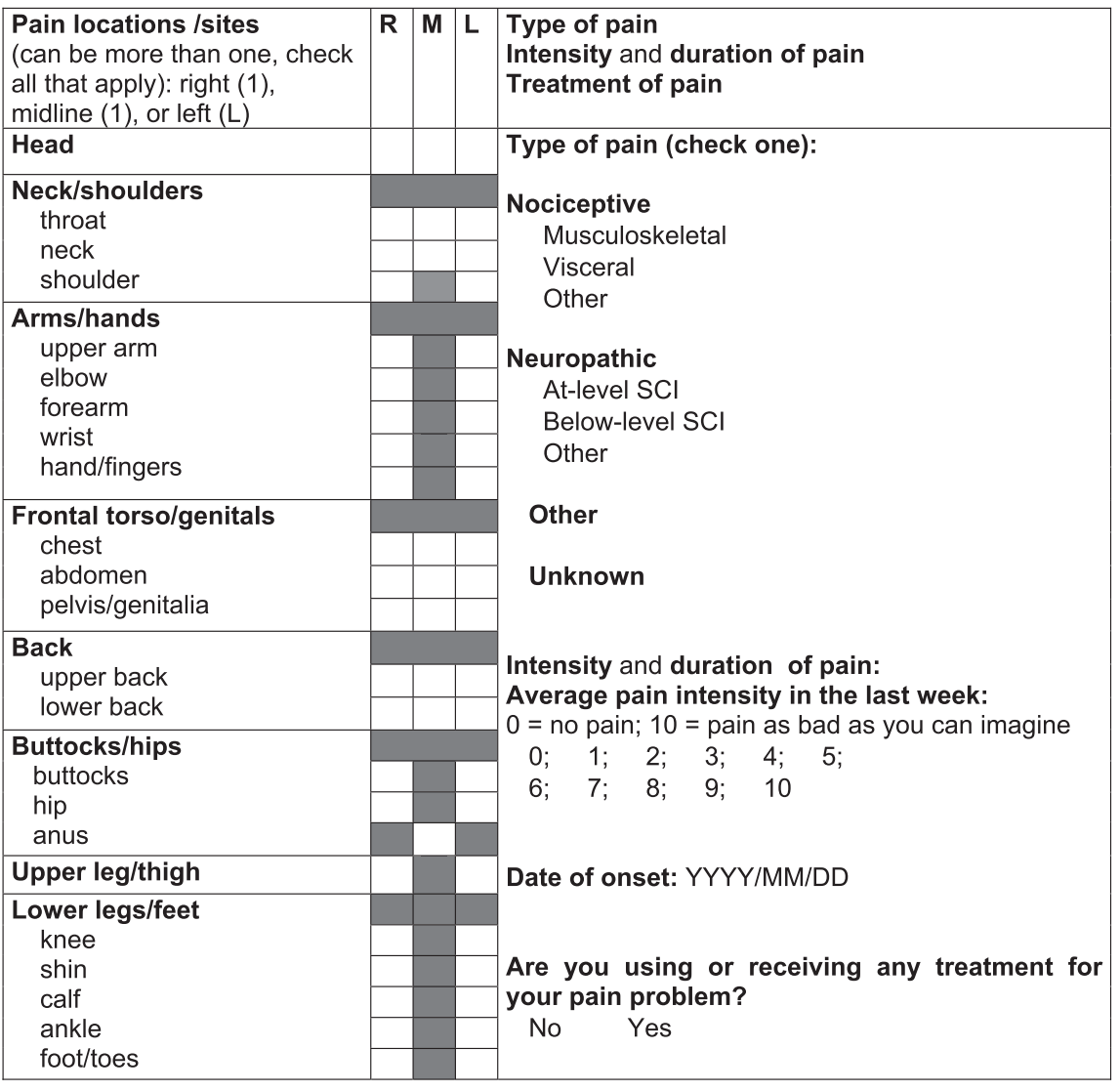

Third worst pain problem:

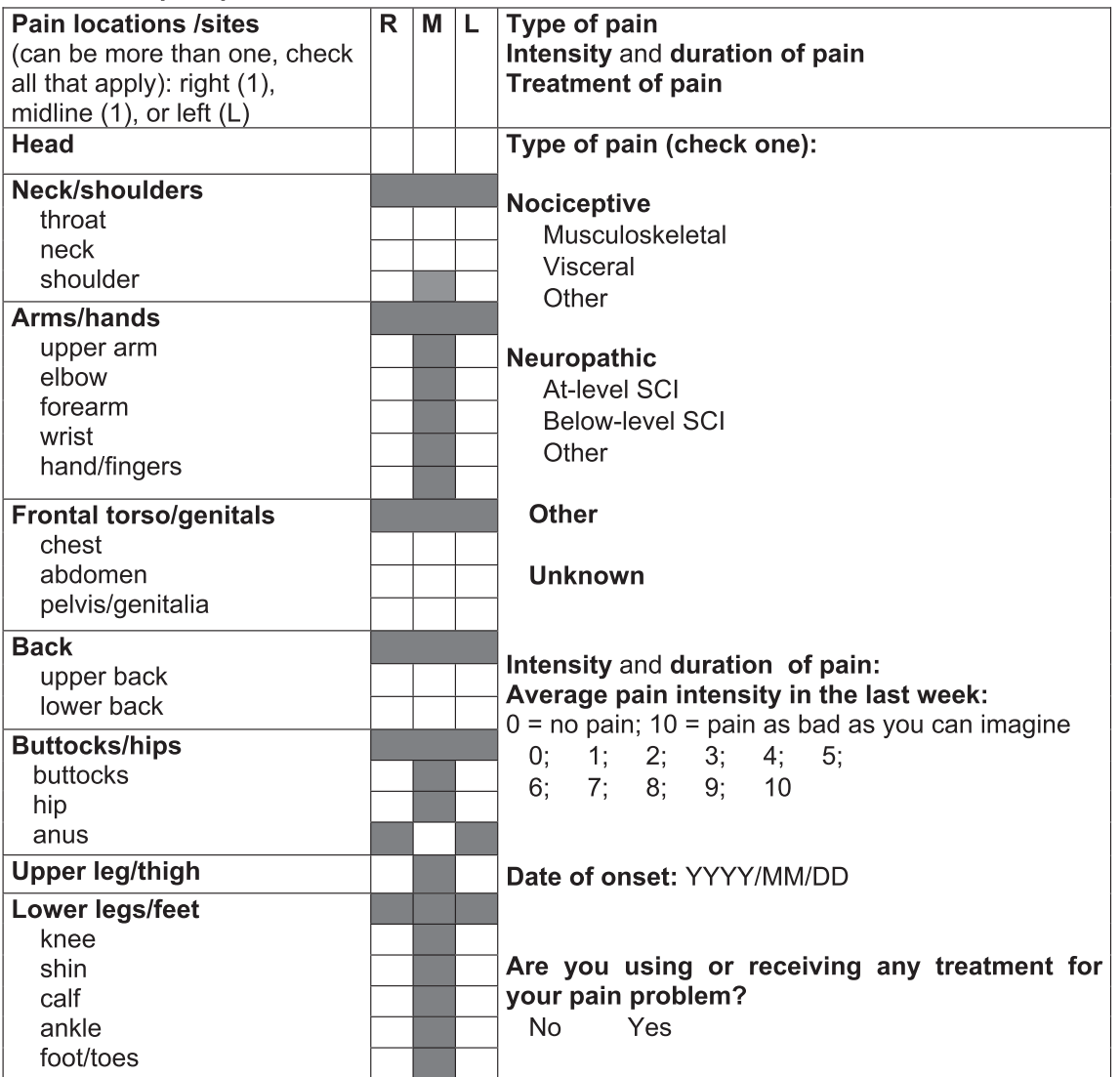

